\title{
Répteis de uma área de Caatinga no Município de Caetés, Agreste Meridional do Estado de Pernambuco, Brasil
}

\section{Rogério Ferreira de Oliveira*, Leandro da Rocha Vieira, Alexandre Gomes Teixeira Vieira}

Laboratório de Zoologia. Universidade de Pernambuco. Campus Garanhuns. Rua Capitão Pedro Rodrigues, 105. São José. Garanhuns-PE. Brasil (CEP 55295-110). *E-mail: biologorogerio87@gmail.com.

Resumo. Devido à carência em dados sobre os répteis da Caatinga, tem crescido estudos acerca desses animais. Motivado pela ausência e necessidade de complementação de informações sobre a herpetofauna do Bioma Caatinga e do Estado de Pernambuco, esse estudo visa a fazer o inventário das espécies de répteis no Vale do Riacho São José, em Caetés, Agreste Meridional do Estado de Pernambuco. Para execução deste estudo foram realizadas buscas ativas no período de setembro de 2012 a janeiro de 2016, para a visualização e registro fotográfico das espécies de répteis existentes na Bacia Hidrográfica do Riacho São José. Foi observado um total de 55 espécies de répteis, divididas em duas ordens, 18 famílias e 39 gêneros. A Família Dipsadidae foi a mais abundante entre os ofídios (62,9\%), entre os lacertílios, a família mais abundante foi Gekkonidae, com $24 \%$ do total. Philodrias nattereri Steindachner, 1870, Tropidurus hispidus (Spix, 1825) e Kynosternon scorpioides (Linnaeus, 1766) figuraram as espécies mais abundantes de Ophidia, Lacertilia e Testudines, respectivamente. Pesquisa e métodos mais criteriosos são necessários para conhecer melhor a fauna de répteis, possibilitando assim a elaboração de planos de manejo e a consequente conservação da biodiversidade local.

Palavras-chave: Inventário; Semiárido; Vale do São José.

Abstract. Reptiles in an area of Caatinga in Caetés, Southern Agreste of Pernambuco State, Brazil. Studies on reptiles of the Caatinga have been carried out due to the lack of data on these animals. Motivated by the absence and need to complement information on the herpetofauna of the Caatinga biome and Pernambuco State, this study aimed to inventory reptile species in the São José Stream Valley, in the Municipality of Caetés, Southern Agreste of Pernambuco State. Active searches were carried out, from September 2012 to January 2016, to visualize and photograph species of reptiles in the São José Stream. Fifty-five reptile species, two orders, eighteen families, and thirty-nine genera were recorded. Dipsadidae (62.9\%) was the most abundant family among the snakes, whereas Gekkonidae (24\%) was the most abundant one among the Lacertilia. Philodrias nattereri Steindachner, 1870, Tropidurus hispidus (Spix, 1825), and Kynosternon scorpioides (Linnaeus, 1766) were the most abundant species of Ophidia, Lacertilia, and Testudines, respectively. Further studies and more rigorous methods
Recebido:

$31 / 03 / 2017$

Aceito:

$14 / 06 / 2017$

Publicado:

30/06/2017

Acesso Aberto

Artigo completo

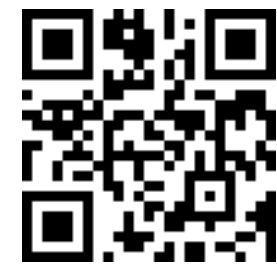

ORCID

(D) 0000-0001-7304-8759

Rogério Ferreira de

Oliveira

(1) 0000-0002-2235-7962

Leandro da Rocha

Vieira

(1) 0000-0003-2644-5764

Alexandre Gomes

Teixeira Vieira 
are needed to understand the reptile fauna better, thus making possible the elaboration of management plans and the consequent local biodiversity conservation.

Keywords: Inventory; Semi-arid; São José Valley.

\section{Introdução}

O conhecimento sobre a fauna brasileira de répteis ainda é incipiente, principalmente, devido à sua enorme diversidade (Martins e Molina, 2010). Essa diversidade ainda é subestimada, devido a inventários insuficientes e ao pequeno número de pesquisadores envolvidos, de modo que, a maioria das informações sobre os répteis brasileiros ainda é preliminar (Rodrigues, 2005).

Em relação à Caatinga, BorgesNojosa e Arzabe (2005) afirmam que ainda não se tem informações eficientes sobre a história natural da maioria das espécies da herpetofauna, de modo a caracterizar suas estratégias reprodutivas e limitações específicas, como dependência a um microhábitat, refúgio ou sítio de desova. Segundo Lema (1969), estudos sobre répteis na Caatinga ainda são muito fragmentados, restritos a áreas específicas. Portanto, o estudo e a conservação da biodiversidade desse bioma constituem um dos maiores desafios do conhecimento científico brasileiro.

Devido à carência em dados sobre os répteis da Caatinga, tem crescido estudos acerca desses animais, ainda assim, grandes áreas desse bioma nunca foram amostradas e novas espécies ainda estão sendo descritas (Borges-Nojosa e Arzabe, 2005). Embora os répteis desse bioma sejam considerados como bem estudados, descobertas inesperadas como o surgimento de novos gêneros e espécies (Rodrigues, 2003, 2005; Fioravante, 2012) reflete quão pouco se sabe em relação aos padrões e processos responsáveis pela evolução e diferenciação da fauna de répteis.

Motivado pela ausência e necessidade de complementação de informações sobre a fauna de répteis da Caatinga e do estado de Pernambuco, esse estudo tem como objetivo fazer o levantamento das espécies de répteis no Vale do Riacho São José, em Caetés, Agreste Meridional de Pernambuco, além de identificar os substratos relacionados à ocorrência dessas espécies e descrever brevemente a importância desses substratos para a ocorrência e distribuição desses animais nessa área.

\section{Materiais e métodos}

O Município de Caetés (coordenadas geográficas representativas 08 46’ 22” S e 36³7’22” O), está localizado na porção Meridional do Planalto da Borborema, a cerca de 240 km da capital Recife, do Estado de Pernambuco, e a cerca de $18 \mathrm{~km}$ do Município de Garanhuns. A área de estudo (Figura 1) está inserida na Bacia Hidrográfica do Riacho São José. A vegetação predominante é de Caatinga, a qual é dividida em áreas de matas secas, florestas semideciduais, savanas, "carrascos", áreas antropizadas e áreas de tensão ecológica. Também há uma variedade em ecossistemas rupestres onde ocorrem cactos e bromélias em grande quantidade ou áreas com subarbustos e arbustos densos. 


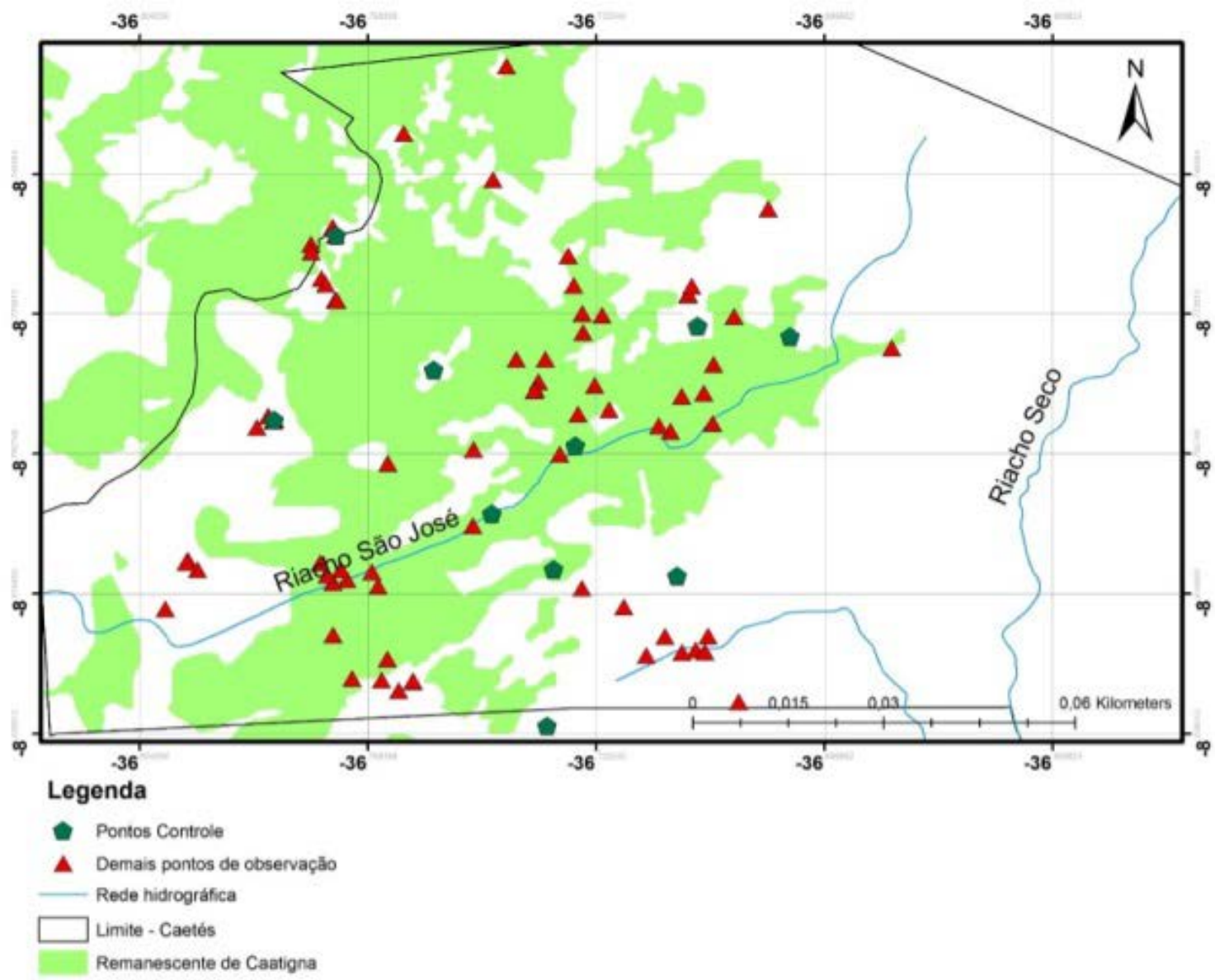

Figura 1. Mapa da área de estudo com pontos de observação.

Para execução deste estudo, foram realizadas buscas ativas no período entre setembro de 2012 e janeiro de 2016, para a visualização e registro fotográfico das espécies de répteis existentes na bacia hidrográfica do riacho São José. Para tanto, foram escolhidos 10 pontos-controle visando à observação mais criteriosa dos répteis. Os mesmos foram visitados periodicamente a cada 30 dias, tanto na estação seca quanto na chuvosa, além de outros 65 pontos de observação situados no entorno dos pontos-controle na área de estudo.

Foram coletados na natureza ou cedidos por populares, animais mortos (Borges-Nojosa e Arzabe, 2005) e demais evidências forenses como esqueletos e peles de animais abatidos (caça cinegética) ou peles descartadas por lacertílios e serpentes após o processo de ecdise. Também foram considerados válidos vestígios, como rastros e pegadas, sabendo da credibilidade desse método de observação para identificação de algumas espécies.

As observações ocorreram durante o dia e também no período noturno em diferentes nichos, como áreas florestais, campos rupestres, pastagens, corpos hídricos e nas proximidades das propriedades rurais. Esses locais foram selecionados com acordo aos nichos favoráveis à ocorrência de determinadas espécies. Não houve instalação de armadilhas de nenhum tipo, os espécimes eventualmente capturados durante as observações foram libertados no local de captura, logo após registro fotográfico. Para identificação das espécies foi consultada a bibliografia especializada em herpetologia 
da Caatinga, o acervo da Coleção Didática de Zoologia (CDZ) da Universidade de Pernambuco, Campus Garanhuns, bem como a colaboração de uma especialista em herpetologia.

\section{Resultados}

Foi registrado um total de 55 espécies de répteis, divididas em duas ordens e dezessete famílias (Tabela 1,
Figura 2). A família Dipsadidae foi a mais representativa entre os ofídios compondo $62,9 \%$ do total de espécies. Entre os lacertílios, a família mais representativa foi Gekkonidae, com 24\% do total. Phylodrias nattereri Steindachner, 1870, Tropidurus hispidus (Spix, 1825) e Kinosternon scorpioides (Linnaeus, 1766), foram as espécies mais abundantes de Ophidia, Lacertilia e Testudines, respectivamente.

Tabela 1. Tabela das espécies de répteis existentes no vale do riacho São José. As espécies endêmicas do bioma Caatinga estão marcadas com asterisco $(*)$.

\begin{tabular}{|c|c|c|c|}
\hline \multirow{2}{*}{$\begin{array}{l}\text { Ordem } \\
\text { Squamata (Ophidia) }\end{array}$} & Família & Espécie & Nome popular \\
\hline & Boidae & Boa constrictor Linnaeus, 1758 & $\begin{array}{l}\text { Jiboia, } \\
\text { Salamanta-boi }\end{array}$ \\
\hline & & $\begin{array}{l}\text { Corallus hortulanus (Linnaeus, } \\
\text { 1758) }\end{array}$ & $\begin{array}{l}\text { Salamanta-de- } \\
\text { parede, } \\
\text { Salamanta- } \\
\text { focinho-de- } \\
\text { cachorro }\end{array}$ \\
\hline & & Epicrates assisi Machado, 1945 & Salamanta \\
\hline & Colubridae & Oxybelis aeneus (Wagler, 1824) & Cobra-de-cipó \\
\hline & & Spilotes pullatus (Linnaeu, 1758) & Papa-ovo \\
\hline & Dipsadidae & $\begin{array}{lll}\begin{array}{l}\text { Apostolepis gaboi } \\
\text { 1992* }\end{array} & \text { Rodrigues, } \\
\end{array}$ & Mata-besta \\
\hline & & Boiruna sertaneja Zaher, 1996 & Cobra-preta \\
\hline & & $\begin{array}{l}\text { Clelia plumbea (Wied-Neuwied, } \\
\text { 1820)* }\end{array}$ & Cobra-preta \\
\hline & & $\begin{array}{l}\text { Erythrolamprus viridis Günther, } \\
1862\end{array}$ & Cobra-verde \\
\hline & & Helicops sp. & Cobra-d'água \\
\hline & & Lygophis dilepis(Cope, 1862) & Corredeira \\
\hline & & $\begin{array}{l}\text { Oxyrhopus trigeminus Duméril, } \\
\text { Bibron and Duméril, } 1854\end{array}$ & Falsa-coral \\
\hline & & $\begin{array}{l}\text { Philodryas aestiva (Duméril, } \\
\text { Bibron and Duméril, 1854) }\end{array}$ & Cobra-verde \\
\hline & & Philodryas agassizii (Jan, 1863) & Corre-campo \\
\hline & & $\begin{array}{ll}\text { Philodryas } & \text { nattereri } \\
\text { Steindachner, } 1870 & \\
\end{array}$ & Corre-campo \\
\hline & & $\begin{array}{l}\text { Philodryas olfersii (Lichtenstein, } \\
\text { 1823) }\end{array}$ & Cobra-verde \\
\hline & & $\begin{array}{l}\text { Philodryas patagoniensis (Girard, } \\
\text { 1857) }\end{array}$ & Cobra-verde \\
\hline & & $\begin{array}{l}\text { Pseudoboa nigra (Duméril, } \\
\text { Bibronand Duméril, 1854) }\end{array}$ & $\begin{array}{l}\text { Cobra-preta, } \\
\text { Caninana }\end{array}$ \\
\hline & & $\begin{array}{lr}\text { Sibynomorphus } & \text { aff. } \\
\text { ventrimaculatus } & \text { (Boulenger, } \\
1885) & \end{array}$ & $\begin{array}{l}\text { Jararaquinha- } \\
\text { dormideira, } \\
\text { Mata-besta, 24- } \\
\text { horas }\end{array}$ \\
\hline
\end{tabular}


Tabela 1. Continuação.

\begin{tabular}{|c|c|c|c|}
\hline Ordem & Família & Espécie & Nome popular \\
\hline \multirow[t]{8}{*}{ Squamata (Ophidia) } & Dipsadidae & $\begin{array}{l}\text { Thamnodynastes } \\
\text { (Linnaeus, 1758) }\end{array}$ & Cobra-rainha \\
\hline & & $\begin{array}{l}\text { Thamnodynastes } \\
\text { Bailey, Thomas and Silva Jr., } \\
2005\end{array}$ & Cobra-de-cipó \\
\hline & & $\begin{array}{l}\text { Thamnodynastes } \\
\text { (Thamnodynastes sp. 2, sensu } \\
\text { Franco \& Ferreira, 2002) }\end{array}$ & $\begin{array}{l}\text { Jararaquinha- } \\
\text { falsa }\end{array}$ \\
\hline & Elapidae & Micrurus sp.* (Merrem, 1820) & $\begin{array}{l}\text { Coral-verdadeira, } \\
\text { 24-horas }\end{array}$ \\
\hline & Viperidae & $\begin{array}{l}\text { Bothrops erythromelas Amaral, } \\
\text { 1923* }\end{array}$ & $\begin{array}{l}\text { Jararaquinha-da- } \\
\text { Caatinga }\end{array}$ \\
\hline & & Bothrops leucurus Wagler, 1824 & Jararaca \\
\hline & & Bothrops neuwiedi Wagler, 1824 & $\begin{array}{l}\text { Jararaca-malha- } \\
\text { de-cascavel }\end{array}$ \\
\hline & & Crotalus durissus Linnaeus, 1758 & Cascavel \\
\hline \multirow[t]{15}{*}{ Squamata (Lacertilia) } & Amphisbaenidae & $\begin{array}{l}\text { Amphisbaena Alba Linnaeus, } \\
1758\end{array}$ & $\begin{array}{l}\text { Cobra-cega, } \\
\text { Cobra-de-duas- } \\
\text { cabeças }\end{array}$ \\
\hline & & $\begin{array}{l}\text { Amphisbaena } \\
\text { Wagler, } 1824\end{array}$ & $\begin{array}{l}\text { Cobra-cega, } \\
\text { Cobra-de-duas- } \\
\text { cabeças }\end{array}$ \\
\hline & & Amphisbaena sp. & $\begin{array}{l}\text { Cobra-cega, } \\
\text { cobra-de-duas- } \\
\text { cabeças }\end{array}$ \\
\hline & Anguidae & $\begin{array}{l}\text { Diploglossus lessonae Peracca, } \\
1890\end{array}$ & Briba \\
\hline & Gekkonidae & $\begin{array}{l}\text { Gymnodactylus geckoides Spix, } \\
1825\end{array}$ & $\begin{array}{l}\text { Lagartixa-de- } \\
\text { espinhos }\end{array}$ \\
\hline & & $\begin{array}{l}\text { Hemidactylus agrius Vanzolini, } \\
1978\end{array}$ & Lagartixa \\
\hline & & $\begin{array}{ll}\text { Hemidactylus } & \text { brasilianus } \\
\text { (Amaral, 1935) } & \\
\end{array}$ & Lagartixa \\
\hline & & $\begin{array}{l}\text { Phyllopezus periosus Rodrigues, } \\
\text { 1986* }\end{array}$ & Lagartixa \\
\hline & & $\begin{array}{l}\text { Phyllopezus pollicaris (Spix, } \\
\text { 1825) }\end{array}$ & Lagartixa-noturna \\
\hline & & $\begin{array}{l}\text { Lygodactylus klugei (H. Smith, } \\
\text { Martin \& Swain, 1977) }\end{array}$ & Calanguinho \\
\hline & Gymnophthalmidae & $\begin{array}{ll}\text { Psilophthalmus } & \text { paeminosus } \\
\text { Rodrigues, 1991* } & \end{array}$ & Briba \\
\hline & & $\begin{array}{ll}\text { Vanzosaura } & \text { rubricauda } \\
\text { (Boulenger, 1902) } & \end{array}$ & $\begin{array}{l}\text { Briba-de- } \\
\text { roçadoou rabo- } \\
\text { de-fogo }\end{array}$ \\
\hline & Iguanidae & Iguana iguana (Linnaeus, 1758) & Iguana, Camaleão \\
\hline & Leiosauridae & $\begin{array}{l}\text { Enyalius bibronii } \text { Boulenger, } \\
1885\end{array}$ & Camaleãozinho \\
\hline & Polychrotidae & Polychrus acutirostris Spix, 1825 & Papa-vento \\
\hline
\end{tabular}


Tabela 1. Continuação.

\begin{tabular}{|c|c|c|c|}
\hline Ordem & Família & Espécie & Nome popular \\
\hline & Scincidae & $\begin{array}{l}\text { Mabuya heathi Schmidt \& Inger, } \\
1951\end{array}$ & Briba \\
\hline & & $\begin{array}{l}\text { Mabuya agmosticha Rodrigues, } \\
\text { 2000* }\end{array}$ & Briba \\
\hline & & $\begin{array}{l}\text { Mabuya macrorhyncha Hoge, } \\
1946\end{array}$ & Briba \\
\hline & Teiidae & Ameiva ameiva (Linnaeus, 1758) & Calango-sardão \\
\hline & & Ameivula ocellifera (Spix, 1825) & Calango-verde \\
\hline & & $\begin{array}{l}\text { Cnemidophorus ocellifer (Spix, } \\
\text { 1825) }\end{array}$ & Calango-grande \\
\hline & & $\begin{array}{l}\text { Tupinambis merianae (Duméril \& } \\
\text { Bibron, 1839) }\end{array}$ & Tiú, Teju \\
\hline & Tropiduridae & $\begin{array}{ll}\text { Tropidurus } & \text { cocorobensis } \\
\text { Rodrigues, } 1987 & \end{array}$ & Lagartixa \\
\hline & & Tropidurus hispidus (Spix, 1825) & Lagartixa \\
\hline & & $\begin{array}{l}\text { Tropidurus semitaeniatus (Spix, } \\
\text { 1825)* }\end{array}$ & Lagartixa* \\
\hline Testudines & Kinosternidae & $\begin{array}{ll}\text { Kinosternon } & \text { scorpioides } \\
\text { (Linnaeus, 1766) } & \\
\end{array}$ & Cágado, Muçuã \\
\hline & Testudinidae & $\begin{array}{l}\text { Chelonoidis carbonaria (Spix, } \\
\text { 1824) }\end{array}$ & Jabuti \\
\hline & Chelidae & $\begin{array}{l}\text { Mesoclemmys } \\
\text { (Luederwaldt,1926)* }\end{array}$ & $\begin{array}{l}\text { Cágado-cabeça- } \\
\text { de-cobra }\end{array}$ \\
\hline Total: & 18 Famílias & $\begin{array}{l}39 \text { Gêneros } \\
55 \text { Espécies }\end{array}$ & \\
\hline
\end{tabular}

\section{Discussão}

O número de répteis encontrado no Vale do Riacho São José é alto se comparado a outras áreas de Caatinga, que dispõem de proteção ambiental, como no Parque Nacional (PARNA) Vale do Catimbau, Pernambuco (Muniz e Santos, 2009), onde foram contabilizadas 30 espécies, no PARNA Raso da Catarina, Bahia (Garda et al., 2013), com 32 espécies, e no PARNA Serra da Capivara, Piauí (Cavalcanti et al., 2014), com 29 espécies.

A fauna de répteis presente no vale do São José apresenta distribuição heterogênea devido à disposição fitofisionômica local, com espécies associadas a áreas florestais, (exemplo das espécies das Famílias Boidae e Iguanidae) e outras adaptadas às áreas abertas ou antropizadas (exemplo das espécies da
Família Teiidae). De acordo com Rodrigues (2005), as espécies da ordem Squamata são, em geral, muito resistentes à fragmentação do hábitat, o que pode explicar a amplitude da ocorrência dos répteis locais. As espécies Clelia plumbea e Corallus hortulanus são as únicas que constam no livro vermelho das espécies ameaçadas de extinção do Brasil, conforme Martins e Molina (2010).

As 27 serpentes listadas no presente estudo correspondem a $24 \%$ das espécies descritas para a Caatinga de acordo com a obra de Guedes, Nogueira e Marques (2014). As serpentes peçonhentas Micrurus sp., Bothrops erythromelas, B. leucurus e Crotalus durissus, encontram-se amplamente distribuídas em toda a área de estudo e do ponto de vista médico, são animais importantes por protagonizarem a maioria dos acidentes ofídicos locais. 

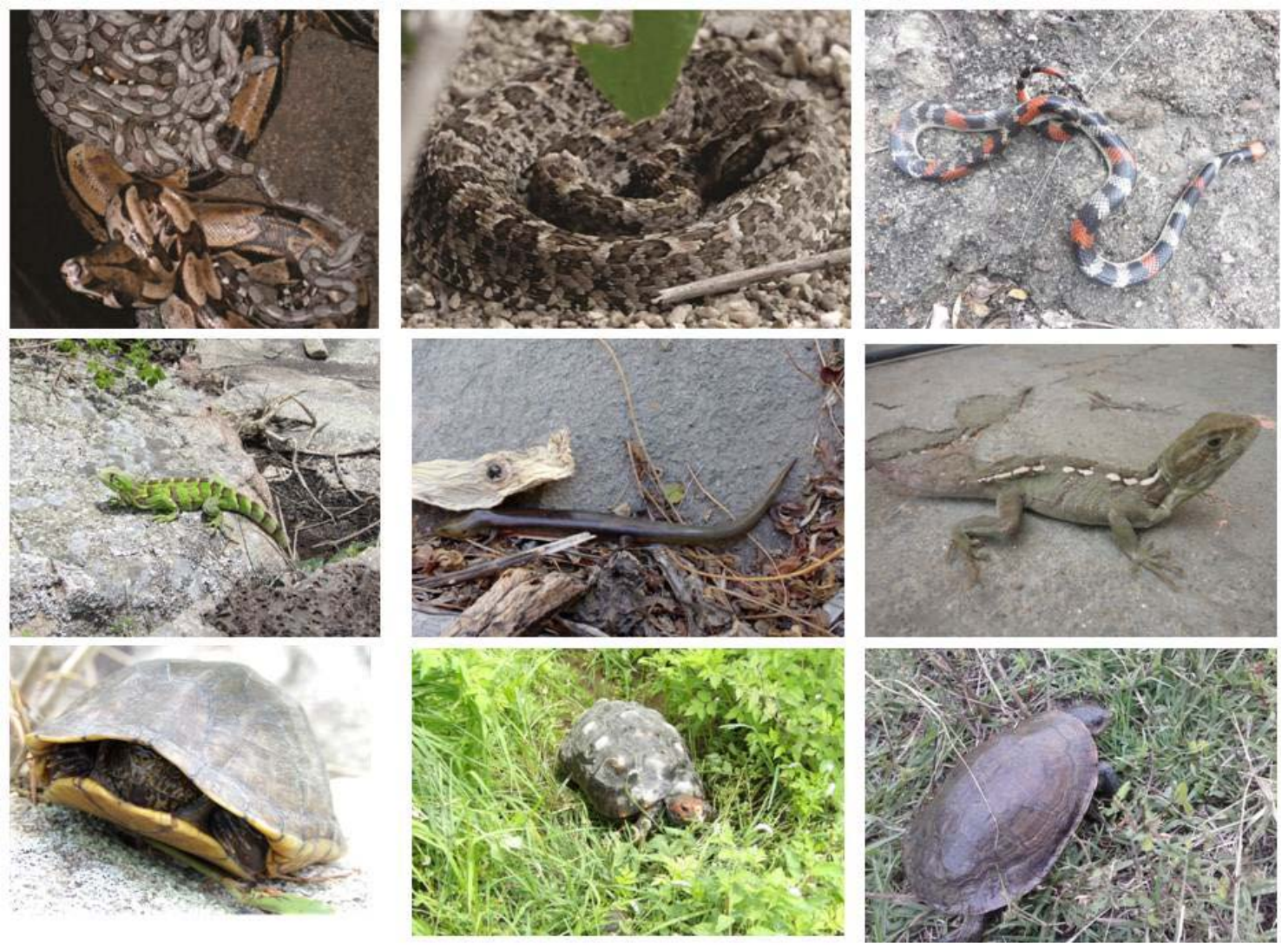

Figura 2. Espécies de répteis observadas no Vale do riacho São José, Caetés, Agreste Meridional de Pernambuco. (A) Boa constrictor Linnaeus, 1758; (B) Bothrops erythromelas Amaral, 1923; (C) Oxyrhopus trigeminus Duméril, Bibron and Duméril, 1854; (D) Iguana iguana (Linnaeus, 1758); (E) Diploglossus lessonae Peracca, 1890; (F) Enyalius bibronii Boulenger,; (G) Kinosternon scorpioides (Linnaeus, 1766); (H) Chelonoidis carbonaria (Spix, 1824); (I) Mesoclemmys tuberculata (Luederwaldt, 1926). Fonte: Banco de dados do Grupo Vale do São José, arquivo de 2016.

A maioria das serpentes da área de estudo é generalista de hábitats, distribuída em áreas arborizadas, carrascos, campos rupestres, em áreas abertas ou com vegetação secundária. Devido ao medo e antipatia, as pessoas acabam exterminando tanto serpentes quanto anfisbenídeos quando encontradas, esse fato corrobora com o evidenciado por Rodrigues (2005).

Em relação aos lacertílios, o número de espécies encontradas no Vale do São José, 25 espécies, equivale a 53\% das espécies descritas para a Caatinga, confrontando Rodrigues (2003). Espécies dos gêneros Ameiva, Tropidurus e Cnemidosphorus foram mais abundantes nesse estudo.

As Famílias Iguanidae, Leiusauridae e Polychrotidae apresentam sensibilidade às ações antrópicas como desmatamentos, por serem dependentes do estrato arbóreo e da serrapilheira abaixo dele, para forrageio e reprodução. Segundo Rodrigues (2005), espécies arborícolas são vulneráveis a destruição de hábitats por não suportarem as altas temperaturas de áreas abertas. Além disso, devido ao sabor da carne (semelhante ao frango) as espécies Iguana iguana e Tupinambis merinae, integram a caça cinegética e a zooterapia local (uso da gordura).

Os lagartos Psilophthalmus paeminosus e Vanzosaura rubricauda apresentam hábitos psamófilos, ao se enterrar no solo, conseguem fugir de predadores, proteger-se do frio e do calor escaldante, como observado por Fioravanti (2012) para espécies dessa família nas 
Dunas do São Francisco. As espécies da Família Scincidae estão relacionadas à microhábitats sob árvores em decomposição e presença de bromeliáceas.

$$
\text { Corroborando com Fioravanti }
$$

(2012), as espécies das Famílias Tropiduridae e Teiidae necessitam de estudos taxonômicos mais criteriosos devido à deficiência em informações sobre elas. Estas famílias constituem grupos complexos e de ampla distribuição em toda a Caatinga. Isto converge com as conclusões de Rodrigues (2005), que que considera que estas famílias constituem um complexo de subespécies, carecendo de uma melhor sistematização. Espécies destas famílias podem estivar durante época de baixas temperaturas ou de escassez de alimento. A estivação ocorre em abrigos rochosos ou em buracos no solo, chegando ao término com o início da estação chuvosa, época de abundância de alimento que ocorre geralmente nos meses de dezembro a março.

Na Caatinga, para a ordem dos Testudines são contabilizadas quatro espécies, segundo Rodrigues (2003). No Vale do São José, são encontradas três dessas espécies, Kinosternon scorpioides, Mesoclemmys tuberculata e Chelonoides carbonaria, corroborando com BorgesNojosa e Arzabe (2005) em estudo nos Municípios de Betânia e Floresta, ambas em Pernambuco. A existência de C. carbonaria no Vale do São José está associada às áreas de vegetação remanescente, onde ela ainda consegue se reproduzir, esta é a espécie mais seriamente ameaçada pela redução e fragmentação de seus hábitats e pela retirada de indivíduos do meio natural. Por vezes acaba aproximando-se das propriedades, onde são capturados e domesticados para rituais mágico-religiosos ou vendidos em feiras livres de cidades próximas (Alves, 2012; Alves et al., 2012a).

Em geral, esses Testudines adaptaram-se às ações antrópicas, se beneficiando da presença de corpos hídricos artificiais utilizados para acumular água das chuvas. A abundância de Kinosternon scorpioides pode está relacionada também ao fato de não integrar a caça cinegética local, diferentemente de Mesoclemmys tuberculata, que além de ser apreciado pelo sabor de sua carne, tem sua gordura utilizada na zooterapia (Alves et al., 2012a).

\section{Considerações finais}

O número de répteis listado para o vale do São José mostrou-se superior ao encontrado em outras áreas, até mesmo as dispõem de proteção ambiental. A presença dessas espécies evidencia a elevada qualidade ambiental da área de estudo e a importância no desenvolvimento de estudos sobre a fauna de répteis dessa localidade, frente à carência de informações para outras áreas de Caatinga em Pernambuco e em todo o bioma.

A diversidade das espécies dessa área está relacionada à grande quantidade e diversidade de hábitats, amplamente distribuídos por toda área de estudo. Essas particularidades permitem a ocorrência de espécies relictuais, as quais exigem condições e nichos adequados para sua ocorrência e sobrevivência.

Pesquisas e métodos mais criteriosos são necessários para conhecer melhor a composição da fauna de répteis, visando fornecer informações ecológicas que nos permita entender a dinâmica populacional dessas espécies. Dessa forma, poderão ser recomendadas ações para efetiva proteção e conservação dessa importante área visando à preservação da fauna de répteis local.

\section{Agradecimentos}

Gostaríamos de agradecer a toda equipe do Laboratório de Zoologia da Universidade de Pernambuco, Campus Garanhuns pelo apoio durante o desenvolvimento dessa pesquisa e as valorosas contribuições da Professora Ednilza Maranhão dos Santos.

\section{Declaração de conflito de interesses}

Os autores declaram não haver conflito de interesses. 


\section{Referências}

Alves, R. R. N. Relationships between fauna and people and the role of ethnozoology in animal conservation. Ethnobiology and Conservation, $1: 2,2012$. https://dx.doi.org/10.15451/ec2012-8-1.2-1-69

Alves, R. R. N.; Gonçalves, M. B. R.; Vieira, W. L. S. Caça, uso e conservação de vertebrados no semiárido brasileiro. Tropical Conservation Science, v. 5, n. 3, p. 394-416, 2012a. Disponível em: $<$ http://tropicalconservationscience.mongabay.c om/content/v5/TCS-2012_Vol_5(3)_394-

416_Alves_et_al.pdf $>$. Acesso em: 23 nov. 2016.

Alves, R. R. N.; Pereira Filho, G. A.; Vieira, K. S.; Souto, W. M. S.; Mendonça, L. E. T.; Montenegro, P. F. G. P.; Almeida, W. O.; Vieira, W. L.S. A zoological catalogue of hunted reptiles in the semiarid region of Brazil. J. Ethnobiol. Ethnomed. $\quad$ 8: 27, 2012b. https://dx.doi.org/10.1186/1746-4269-8-27

Borges-Nojosa, D. M.; Santos, E. M. Herpetofauna da área de Betânia e Floresta, Pernambuco. In: Araújo, F. S.; Rodal, M. J. N.; Barbosa, M. R. V. Análise das variações da biodiversidade do bioma Caatinga: suporte a estratégias regionais de conservação. Brasília: Ministério do Meio Ambiente, 2005.

Cavalcanti, L. B. Q.; Costa, T. B.; Colli, G. R.; Costa, G. C.; França, F. G. R.; Mesquita, D. O.; Palmeira, C. N. S.; Pelegrin, N.; Soares, A. H. B.; Tucker, D. B.; Garda, A. A. Herpetofauna of protected areas in the Caatinga II: Serra da Capivara National Park, Piauí, Brazil. Check List, v. 10, n. 1, p. 18-27, 2014. Disponível em: <http://www.checklist.org.br/ getpdf?SL042-13>. Acesso em: 23 nov. 2016.

Fioravante, C. Dunas do Rio São Francisco: a Galápagos brasileira. Revista Fapesp, n. 8, p. 93-97, 2012. Disponível em: $<$ http://revistapesquisa.fapesp.br/wpcontent/uploads/2012/08/94-97_lagartos.pdf>. Acesso em: 23 nov. 2016.
Garda, A. A.; Costa, T. B.; Santos-Silva, C. R.; Mesquita, D. O.; Faria, R. G.; Conceição, B. M.; Silva, I. R. S.; Ferreira, A. S.; Rocha, S. M.; Palmeira, C. N. S.; Rodrigues, R.; Ferrari, S. F.; Torquato, S. Herpetofauna of protected areas in the Caatinga I: Raso da Catarina Ecological Station (Bahia, Brazil). Check List, v. 9, n. 2, p. 405-414, 2013. Disponível em: <http://www.checklist.org.br/getpdf?SL11512>. Acesso em: 23 nov. 2016.

Guedes, T. B.; Nogueira, C.; Marques, O. A. Diversity, natural history, and geographic distribution of snakes in the Caatinga, Northeastern Brazil. Zootaxa, v. 3862, n. 1, p. 1-93, 2014. https://dx.doi.org/10.11646/zootaxa. 3863.1.1

Lema, T. Apreciação geral sumária sobre os répteis do Estado de Pernambuco e adjacências. Recife: s. ed., 1969.

Martins, M.; Molina, F. B. Panorama geral dos répteis ameaçados do Brasil. In: Machado, A. B. M.; Drumund, G. M.; Paglia, A. P. Livro vermelho da fauna brasileira ameaçada de extinção. Brasília: Ministério do Meio Ambiente, 2008. v. 1. (Biodiversidade, 19).

Muniz, S. L. S.; Santos, E. M. Répteis do Vale do Catimbau, Buíque/PE. Anais do Jornada de Pesquisa, Ensino e Extensão - JEPEX, 2009. Disponível em: <http://www.eventosufrpe. com.br/jepex2009/cd/resumos/R0395-1.pdf>.

Acesso em: 29 dez. 2015.

Rodrigues, M. T. U. Conservação dos répteis brasileiros: os desafios para um país megadiverso. Megadiversidade, v. 1. n. 1, p. 87-94, 2005.

Rodrigues, M. T. U. Herpetofauna da Caatinga. In: Leal, I. R.; Tabarelli, M.; Silva, J. M. C. (Eds.). Ecologia e conservação da Caatinga. Recife: Ed. Universitária UFPE, 2003. 